\section{Kinematic controllability and motion planning for the} snakeboard*

\author{
Francesco Bullo ${ }^{\dagger} \quad$ Andrew D. Lewis ${ }^{\ddagger}$
}

$14 / 12 / 2001$

Last updated: $14 / 03 / 2004$

\section{Abstract}

The snakeboard is shown to possess two decoupling vector fields, and to be kinematically controllable. Accordingly, the problem of steering the snakeboard from a given ically controllable. Accordingly, the problem of steering the snakeboard from a given
configuration at rest to a desired configuration at rest is posed as a constrained static configuration at rest to a desired configuration at rest is posed as a constrained static
nonlinear inversion problem. An explicit algorithmic solution to the problem is provided, and its limitations are discussed. An ad hoc solution to the nonlinear inversion problem is also exhibited.

Keywords. nonholonomic mechanics, trajectory generation

AMS Subject Classifications. 70H03, 70005

\section{Introduction}

The snakeboard was first studied by Lewis, Ostrowski, Murray, and Burdick [1994] as an interesting mechanical control system with nonholonomic constraints. In this initial paper, various "gaits" were observed for the snakeboard which suggested that the system should be locally controllable. These gaits used periodic controls, and gave trajectories which, while interesting, are too complicated to use as tools for motion planning. The snakeboard was further studied by Ostrowski [1995] using general techniques presented by Bloch, Krishnaprasad, Marsden, and Murray [1996] for understanding the dynamics of mechanical systems with symmetry and constraints. Ostrowski in his thesis gave the first proof for the local controllability of the snakeboard [see also Ostrowski and Burdick 1997]. In [Ostrowski, Desai, and Kumar 2000] the authors investigate numerically generated optimal trajectories for the snakeboard. Until now, the goal of analytically determining steering controls for the snakeboard has not been achieved.

This goal is accomplished in this paper via the notion of kinematic controllability introduced by Bullo and Lynch [2001]. This notion relies on the finding of a large enough

\section{*To appear in IEEE Transactions on Robotics and Automation} Champaign, Urbana-Champaign, IL 61801, U.S.A.

Email: bullo@uiuc.edu, URL: http://motion.csl.uiuc.edu/ "bullo/

Research supported in part by NSF grants IIS-0118146 and CMS-0100162.

¥Assistant Professor, Department of Mathematics and Statistics, Queen's University, Kingston, ON K7L 3N6, CANADA

Theensu.ca, URL: http://www.mast.queensu.ca/ /andrew/

part by a grant from the Natural Sciences and Engineering Research Council of Canada. collection of so-called "decoupling vector fields." These are vector fields whose integral curves can be followed with an arbitrary parameterisation. The idea is that if a system has a collection of such vector fields whose involutive closure has maximal rank, then one can achieve controllability only by following concatenations of these integral curves starting and ending each integral curve at rest. In this paper we show that the snakeboard is kinematically controllable (this follows from the calculations of Lewis [2000] provided one is aware of the idea of kinematic controllability). Note that this requires the slight extension of the original presentation of Bullo and Lynch [2001] to general affine connections. Also, the computations are facilitated by the use of pseudo-velocities for mechanical systems with constraints as in [Bullo and Žefran 2002]. The motion primitives we propose here do not generate nonholonomic momentum. This is in contrast to, for example, the work in [Ostrowski 2000, Ostrowski, Desai, and Kumar 2000].

The layout of the paper is as follows. In Section 2 we introduce the model for the snakeboard, and generate the objects useful for determining the kinematic controllability of the snakeboard. Kinematic controllability -in general, and in particular for the snakeboard - is discussed in Section 3. In Section 4, a motion planning problem for the snakeboard is posed, and the existence of an explicit solution demonstrated. We also indicate that significant improvements are possible to the explicit solution we give, although a general methodology, especially in the presence of constraints on the actuator angles for the snakeboard, appears to be difficult to obtain.

\section{Modelling of the snakeboard}

In this section we provide the model we use for the snakeboard. First we provide the basic problem data - the configuration space, the inertia matrix, the constraints, and the input forces. Then we use this to provide the equations of motion using the methodology of Bullo and Zefran [2002]. This methodology is a simplification of the presentation in the paper [Lewis 2000], and makes it possible to compactly write the equations of motion for the snakeboard, as well as understand the structure of these equations in the context of affine differential geometry. As we shall see in Section 3, it is in the setting of affine differential geometry that the main ideas for motion planning are derived.

2.1. The data for the snakeboard model. The snakeboard is a toy on which the rider places her two feet on platforms joined by a rigid coupling bar as in Figure 1. We model the system as in Figure 2. Note that we require the angle that the front and back wheels of the snakeboard make with the coupler to be equal. Thus the snakeboard has a five-dimensional configuration space, and we use the coordinates $(x, y, \theta, \psi, \phi)$ as in Figure 2. We think of the configuration space as being $Q=S E(2) \times \mathbb{S} \times \mathbb{S}$. The system possess a Riemannian metric defined by the inertia matrix

$$
M=\left[\begin{array}{ccccc}
m & 0 & 0 & 0 & 0 \\
0 & m & 0 & 0 & 0 \\
0 & 0 & J+J_{r}+J_{w} & J_{r} & 0 \\
0 & 0 & J_{r} & J_{r} & 0 \\
0 & 0 & 0 & 0 & J_{w}
\end{array}\right] .
$$




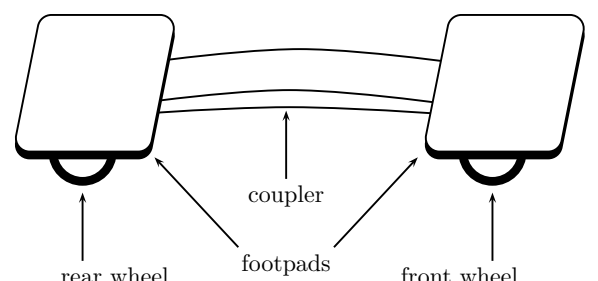

Figure 1. The snakeboard toy

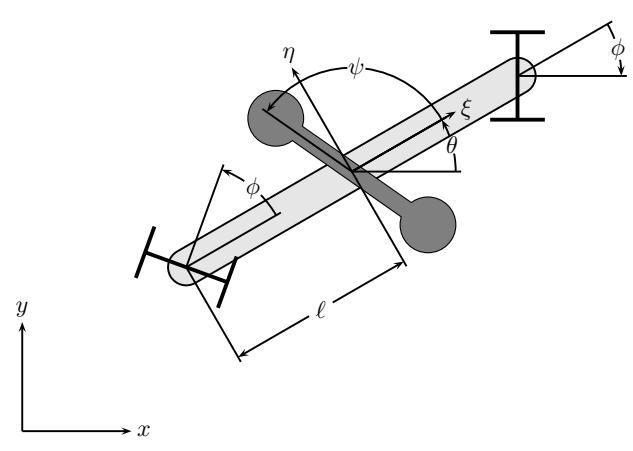

Figure 2. The model for the snakeboard

Here $m$ is the total mass of the snakeboard, $J$ the moment of inertia of the coupler about its centre, $\frac{1}{2} J_{w}$ the moment of inertia of the wheels about their point of rotation, and $J_{r}$ the inertia of the rotor about its point of rotation. We note that certain simplifications are made in this model, but make no further comment on this here.

The other essential ingredient in the snakeboard model is the constraints. It is convenient to introduce the vector fields

$$
V_{x}=\cos \theta \frac{\partial}{\partial x}+\sin \theta \frac{\partial}{\partial y}, \quad V_{y}=-\sin \theta \frac{\partial}{\partial x}+\cos \theta \frac{\partial}{\partial y} .
$$

One may readily show [see Bullo and Žefran 2002, Lewis 2000] that the velocities allowed by the constraints are linear combinations of the three vector fields

$$
\begin{gathered}
X_{1}=\ell \cos \phi V_{x}-\sin \phi \frac{\partial}{\partial \theta}, \\
X_{2}^{\prime}=\frac{\partial}{\partial \psi}, \quad X_{3}=\frac{\partial}{\partial \phi} .
\end{gathered}
$$

In order to use the procedure of Bullo and Žefran [2002], as we intend to do, one must determine a basis of asmissible velocities that are orthogonal with respect to the inner product defined by the inertia matrix (2.1). One may readily check that $X_{3}$ is orthogonal to both $X_{1}$ and $X_{2}^{\prime}$. Thus it suffices to ensure modify $X_{2}^{\prime}$ in such a way that it is orthogonal to $X_{1}$ while still being orthogonal to $X_{3}$. We may do that by defining

$$
X_{2}=c_{1}^{-1}\left(\left\langle X_{1}, X_{1}\right\rangle X_{2}^{\prime}-\left\langle X_{1}, X_{2}^{\prime}\right\rangle X_{1}\right),
$$

for any nonzero function $c_{1}$ on $Q$. It turns out to be convenient to take

$$
c_{1}(\phi)=m \ell^{2} \cos ^{2} \phi+\left(J+J_{r}+J_{w}\right) \sin ^{2} \phi .
$$

In [Ostrowski and Burdick 1997] the assumption is made that $J+J_{r}+J_{w}=m \ell^{2}$, giving a corresponding simplification for $c_{1}$. The resulting computations give

$$
X_{2}=\frac{J_{r} \ell \cos \phi \sin \phi}{c_{1}(\phi)} V_{x}-\frac{J_{r} \sin ^{2} \phi}{c_{1}(\phi)} \frac{\partial}{\partial \theta}+\frac{\partial}{\partial \psi} .
$$

Motivated by this we define

$$
a(\phi)=\frac{J_{r} \ell \cos \phi \sin \phi}{c_{1}(\phi)}, \quad b(\phi)=\frac{J_{r} \sin ^{2} \phi}{c_{1}(\phi)} .
$$

Therefore, to summarise, we have provided three vector fields,

$$
\begin{gathered}
X_{1}=\ell \cos \phi V_{x}-\sin \phi \frac{\partial}{\partial \theta}, \\
X_{2}=a(\phi) V_{x}-b(\phi) \frac{\partial}{\partial \theta}+\frac{\partial}{\partial \psi}, \quad X_{3}=\frac{\partial}{\partial \phi} .
\end{gathered}
$$

orthogonal with respect to the inner product defined by the inertia matrix (2.1), that span the set of admissible velocities. Let us denote by $\mathrm{D}$ the distribution spanned by these vector fields. Thus $\mathrm{D}_{q} \subset T_{q} Q$ is the set of admissible velocities at the configuration $q \in Q$.

Finally, we consider the forces used to actuate the snakeboard. Mechanically, the input forces are torques applied to the wheels and to the rotor. Thought of as one-forms, as forces ought to be, the forces are represented by

$$
F^{\psi}=\mathrm{d} \psi, \quad F^{\phi}=\mathrm{d} \phi .
$$

In the equations of motion that we consider in the subsequent section, it is actually not these one-forms which come up, but rather vector fields which we construct from these one forms. First we must convert the one-forms to vector fields by multiplying by the inverse of the inertia matrix $M$. The resulting vector fields are then

$$
Y_{\psi}^{\prime}=\frac{1}{J+J_{w}}\left(\frac{J+J_{r}+J_{w}}{J_{r}} \frac{\partial}{\partial \psi}-\frac{\partial}{\partial \theta}\right), \quad Y_{\phi}^{\prime}=\frac{1}{J_{w}} \frac{\partial}{\partial \phi} .
$$

To use these vector fields in the equations of motion in the next section, we must further orthogonally project them onto the D. Let us denote by $P: T Q \rightarrow T Q$ the orthogonal projection onto D. Thus

$$
Y_{\psi}=P\left(Y_{\psi}^{\prime}\right)=\sum_{i=1}^{3} \frac{\left\langle Y_{\psi}^{\prime}, X_{i}\right\rangle}{\left\|X_{i}\right\|^{2}} X_{i}
$$


where $\langle\cdot, \cdot\rangle$ and $\|\cdot\|$ are the inner product and norm relative to the inertia matrix $M$. A similar expression holds, of course, for $Y_{\phi}=P\left(Y_{\phi}^{\prime}\right)$. The computations give

where

$$
Y_{\psi}=\frac{c_{1}(\phi)}{J_{r} c_{2}(\phi)} X_{2}, \quad Y_{\phi}=\frac{1}{J_{w}} X_{3},
$$

$$
c_{2}(\phi)=m \ell^{2} \cos ^{2} \phi+\left(J+J_{w}\right) \sin ^{2} \phi .
$$

2.2. The snakeboard equations of motion. Classically, one would write the equations of motion of the system using Lagrange multipliers. This was worked out in [Lewis, Ostrowski, Murray, and Burdick 1994]. In [Lewis 2000] the snakeboard equations of motion are presented as geodesic equations for an affine connection not compatible with a Riemanare presented as geodesic equations for an affine connection not compatible with a Rieman-
nian metric. This method originates with Synge [1928], although the work of Lewis was motivated by the work of Bloch and Crouch which led to the paper [Bloch and Crouch 1998]. We denote by $P^{\perp}: T Q \rightarrow T Q$ the orthogonal projection onto $\mathrm{D}^{\perp}$, the latter being the orthogonal complement of $\mathrm{D}$ relative to the inner product defined by $M$. Lewis shows that the equations for the snakeboard may be written as

$$
\tilde{\nabla}_{\dot{q}} \dot{q}=u_{\psi} Y_{\psi}+u_{\phi} Y_{\phi},
$$

where the affine connection $\tilde{\nabla}$ is the affine connection defined by

$$
\tilde{\nabla}_{X} Y=\nabla_{X} Y+\left(\nabla_{X} P^{\perp}\right)(Y)
$$

Here $\nabla$ is the regular Levi-Civita affine connection associated with $M$. Lewis [1998] shows that if $Y$ takes values in the distribution $\mathrm{D}$ then we have $\tilde{\nabla}_{X} Y=P\left(\nabla_{X} Y\right)$. This allows one to simplify the computation of the covariant derivative of vector fields taking values in D. We shall be interested primarily in knowing the covariant derivative $\tilde{\nabla}_{X} Y$ when both $X$ and $Y$ take values in D. In this case, it suffices to consider $X, Y \in\left\{X_{1}, X_{2}, X_{3}\right\}$. Bullo and Žefran [2002] show that one has, for $\alpha, \beta, \gamma \in\{1,2,3\}$,

$$
\left\langle\tilde{\nabla}_{X_{\alpha}} X_{\beta}, X_{\gamma}\right\rangle=\left\langle\nabla_{X_{\alpha}} X_{\beta}, X_{\gamma}\right\rangle
$$

so that one can write

$$
\tilde{\nabla}_{X_{\alpha}} X_{\beta}=\sum_{\gamma=1}^{3} \tilde{\Gamma}_{\alpha \beta}^{\gamma} X_{\gamma}
$$

where the generalised Christoffel symbols are given by

$$
\tilde{\Gamma}_{\alpha \beta}^{\gamma}=\frac{1}{\left\|X_{\gamma}\right\|^{2}}\left\langle\nabla_{X_{\alpha}} X_{\beta}, X_{\gamma}\right\rangle .
$$

Note that one can define these generalised Christoffel form a full orthogonal basis of vector fields, or even for a general basis of vector fields. However, since we are interested in vector fields taking values in $\mathrm{D}$, we restrict ourselves to these computations. In this case, we determine that the nonzero Christoffel symbols are

$$
\begin{gathered}
\tilde{\Gamma}_{13}^{1}=\frac{\left(J+J_{r}+J_{w}-m \ell^{2}\right) \cos \phi \sin \phi}{c_{1}(\phi)} \\
\tilde{\Gamma}_{23}^{1}=\frac{J_{r} m \ell^{2} \cos \phi}{c_{1}(\phi)^{2}}, \quad \tilde{\Gamma}_{13}^{2}=-\frac{2 m \ell^{2} \cos \phi}{c_{2}(\phi)}, \quad \tilde{\Gamma}_{23}^{2}=-\frac{J_{r} m \ell^{2} \cos \phi \sin \phi}{c_{1}(\phi) c_{2}(\phi)} .
\end{gathered}
$$

To provide the equations of motion for constrained systems, Bullo and Žefran [2002] use the pseudo-velocities $\left(v^{1}, v^{2}, v^{3}\right)$ defined so that

$$
\dot{q}=\sum_{\alpha=1}^{3} v^{\alpha} X_{\alpha} .
$$

In terms of these pseudo-velocities, the equations of motion for the snakeboard are

$$
\begin{aligned}
\dot{x} & =\ell \cos \phi \cos \theta v^{1}+\frac{J_{r} \ell \cos \phi \sin \phi \cos \theta}{c_{1}(\phi)} \dot{\psi} \\
\dot{y} & =\ell \cos \phi \sin \theta v^{1}+\frac{J_{r} \ell \cos \phi \sin \phi \sin \theta}{c_{1}(\phi)} \dot{\psi} \\
\dot{\theta} & =-\sin \phi v^{1}-\frac{J_{r} \sin ^{2} \phi}{c_{1}(\phi)} \dot{\psi} \\
\dot{v}^{1} & =-\frac{\left(J+J_{r}+J_{w}-m \ell^{2}\right) \cos \phi \sin \phi}{c_{1}(\phi)} v^{1} \dot{\phi}-\frac{J_{r} m \ell^{2} \cos \phi}{c_{1}(\phi)^{2}} \dot{\psi} \dot{\phi} \\
\ddot{\psi} & =\frac{2 m \ell^{2} \cos \phi}{c_{2}(\phi)} v^{1} \dot{\phi}+\frac{J_{r} m \ell^{2} \cos \phi \sin \phi}{c_{1}(\phi) c_{2}(\phi)} \dot{\phi} \dot{\psi}+\frac{c_{1}(\phi)}{J_{r} c_{2}(\phi)} u_{\psi} \\
\ddot{\phi} & =\frac{1}{J_{w}} u_{\phi}
\end{aligned}
$$

There appear to be fewer equations here than there should be. This is a result of an elimination which occurs because of the equalities $v^{2}=\dot{\psi}$ and $v^{3}=\phi$

2.1 REMARK: We make the observation that the "nonholonomic momentum" for the snakeboard is essentially the momentum conjugate to the pseudo-velocity $v^{1}$. That the forces do not appear in the equation for $v^{1}$ provides the proper motivation for the assumption by Ostrowski [1995] that the variables $(\psi, \phi)$ for the snakeboard are directly actuated. To do this properly is a short computation, but in essence the reason is as we have noted here. In a related vein, we remark that the motion primitives of Section 4 have the property that they do not alter the nonholonomic momentum. Such momentum preserving motions were also studied by Ostrowski in his dissertation.

\section{Kinematic controllability}

In this section we assemble the data and computations from Section 2 to show that the snakeboard is, in the terminology of Bullo and Lynch [2001], kinematically controllable. This relies on the notion of a decoupling vector field, and we review this, in the general affine connection setting, in Section 3.1. The general analysis is applied to the snakeboard in Section 3.2.

3.1. A review of kinematic controllability. In this section we consider a general control system corresponding to an affine connection $\nabla$ on a manifold $Q$ and $m$ input vector fields $\left\{Y_{1}, \ldots, Y_{m}\right\}$ on $Q$ :

$$
\nabla_{\dot{q}} \dot{q}=\sum_{a=1}^{m} Y_{a} u_{a}
$$


What we present here comes essentially from [Bullo and Lynch 2001], with this work being motivated by [Lynch, Shiroma, Arai, and Tanie 2000]. We let $Y$ be the distribution spanned by the vector fields $\left\{Y_{1}, \ldots, Y_{m}\right\}$, and we suppose this distribution to be regular. A vector field $X$ on $Q$ is a decoupling vector field for (3.1) if for every integral curve $t \mapsto c(t)$ for $X$ and for every reparameterisation $t \mapsto \tau(t)$ for $c$, there exists a control $t \mapsto u(t)$ with the property that $c \circ \tau$ satisfies (3.1) with the control $u$. The system (3.1) is kinematically controllable if there exists a collection of decoupling vector fields $\left\{X_{1}, \ldots, X_{s}\right\}$ so that their involutive closure has maximal rank at each point $q \in Q$. We note that this notion of kinematic controllability strictly generalises the notion of "reducibility" of a system of the form (3.1) discussed by Lewis [1999]. The idea behind a kinematically controllable system is that one uses concatenations of integral curves for decoupling vector field to accomplish an objective. In doing this, it is important that one begin and end each segment of the concatenated curve with zero velocity.

Let us look at conditions which determine when a given vector field is a decoupling vector field. This result is essentially given by Bullo and Lynch [2001], although they only consider the case when $\nabla$ is the Levi-Civita connection for a Riemennian metric. The result for general affine connections is also stated by Cortés, Martínez, and Bullo [2002].

3.1 Proposition: A vector field $X$ is a decoupling vector field for the system (3.1) if and only if

(i) $X(q) \in \mathrm{Y}_{q}$ for each $q \in Q$ and

(ii) $\nabla_{X} X(q) \in Y_{q}$ for each $q \in Q$.

Proof: First suppose that (i) and (ii) hold. To show that a vector field $X$ is a decoupling vector field, it suffices to show that for any function $f: Q \rightarrow \mathbb{R}$ and any integral curve $c$ for $f X$, there exists a control $u$ so that $c$ and $u$ satisfy (3.1). Letting $f$ and $c$ be so chosen, we have

$$
\begin{aligned}
\nabla_{c^{\prime}(t)} c^{\prime}(t) & =\nabla_{f X} f X(c(t)) \\
& =f^{2}(c(t)) \nabla_{X} X(c(t))+f(c(t))\left(\mathscr{L}_{X} f(c(t))\right) X(c(t)) .
\end{aligned}
$$

Now using (i) and (ii) we have

$$
X(c(t)), \nabla_{X} X(c(t)) \in \mathrm{Y}_{c(t)} .
$$

Therefore, there exists $t \mapsto u(t)$ so that

$$
f^{2}(c(t)) \nabla_{X} X(c(t))+f(c(t))\left(\mathscr{L}_{X} f(c(t))\right) X(c(t))=u^{a}(t) Y_{a}(c(t)),
$$

and this shows that $X$ is a decoupling vector field.

Now suppose that $X$ is a decoupling vector field and let $q \in Q$. Let $t \mapsto c(t)$ be the integral curve of $X$ through $q$ and suppose that $c(0)=q$. Define two reparameterisations, $\tau_{1}$ and $\tau_{2}$, of $c$ with the following properties:

1. $\tau_{1}(0)=0, \tau_{1}^{\prime}(0)=1$, and $\tau_{1}^{\prime \prime}(0)=0$;

2. $\tau_{2}(0)=0, \tau_{2}^{\prime}(0)=1$, and $\tau_{2}^{\prime \prime}(0)=1$
Let $c_{1}=c \circ \tau_{1}$ and $c_{2}=c \circ \tau_{2}$. We then have

$$
\begin{aligned}
\nabla_{c_{1}^{\prime}(t)} c_{1}^{\prime}(t) & =\nabla_{c^{\prime}(\tau(t)) \tau_{1}^{\prime}(t)} c^{\prime}(\tau(t)) \tau_{1}^{\prime}(t) \\
& =\left(\tau_{1}^{\prime}(t)\right)^{2} \nabla_{c^{\prime}\left(\tau_{1}(t)\right)} c^{\prime}\left(\tau_{1}(t)\right)+\tau_{1}^{\prime \prime}(t) c^{\prime}\left(\tau_{1}(t)\right) .
\end{aligned}
$$

Evaluating this at $t=0$ gives

$$
\left.\nabla_{c_{1}^{\prime}(t)} c_{1}^{\prime}(t)\right|_{t=0}=\nabla_{X} X(q)
$$

Similarly for $c_{2}$ we have

$$
\left.\nabla_{c_{2}^{\prime}(t)} c_{2}^{\prime}(t)\right|_{t=0}=\nabla_{X} X(q)+X(q) .
$$

Since we are assuming that $X$ is a decoupling vector field for the system we then have

$$
\nabla_{X} X(q)=u_{1}^{a} Y_{a}(q), \quad \nabla_{X} X(q)+X(q)=u_{2}^{a} Y_{a}(q)
$$

for some $u_{1}, u_{2} \in \mathbb{R}^{m}$. This then clearly implies that $\nabla_{X} X(q), X(q) \in \mathrm{Y}_{q}$, as in the statement of the proposition.

It is possible to determine the controls for the system (3.1) needed to move along a decoupling vector field with a specified reparameterisation.

3.2 Proposition: Let $X$ be a decoupling vector field for the system (3.1), let $t \mapsto c(t)$ be an integral curve for $X$ and let $t \mapsto \tau(t)$ be a reparameterisation for $c$. If $t \mapsto u(t) \in \mathbb{R}^{m}$ is defined by

$$
u^{a}(t) Y_{a}(c \circ \tau(t))=\left(\tau^{\prime}(t)\right)^{2} \nabla_{X} X(c \circ \tau(t))+\tau^{\prime \prime}(t) X(c \circ \tau(t)),
$$

then co $\tau$ satisfies (3.1) with control $u$.

Proof: Since $c$ is an integral curve for $X, c^{\prime}(t)=X(c(t))$. We therefore have

$$
\begin{aligned}
\nabla_{(c \circ \tau)^{\prime}(t)}(c \circ \tau)^{\prime}(t) & =\left(\tau^{\prime}(t)\right)^{2} \nabla_{c^{\prime}(\tau(t))} c^{\prime}(\tau(t))+\tau^{\prime \prime}(t) c^{\prime}(\tau(t)) \\
& =\left(\tau^{\prime}(t)\right)^{2} \nabla_{X} X(c \circ \tau(t))+\tau^{\prime \prime}(t) X(c \circ \tau(t)) .
\end{aligned}
$$

The result now follows since $X$ is a decoupling vector field so that

$$
\left(\tau^{\prime}(t)\right)^{2} \nabla_{X} X(c \circ \tau(t))+\tau^{\prime \prime}(t) X(c \circ \tau(t)) \in \mathrm{Y}_{c} \circ \tau(t) .
$$

3.2. Kinematic controllability of the snakeboard. We now apply the notions of decoupling vector fields and kinematic controllability to the snakeboard. First we show that the vector fields $X_{2}$ and $X_{3}$ defined in Section 2.1 are decoupling vector fields. Indeed, since in Section 2.1 we showed that the input vector field $Y_{\psi}$ is a multiple of $X_{2}$ and that the input vector field $Y_{\phi}$ is a multiple of $X_{3}$, it follows that both $X_{2}$ and $X_{3}$ take values in Y. Furthermore, we compute

$$
\nabla_{X_{2}} X_{2}=\sum_{\alpha=1}^{3} \tilde{\Gamma}_{22}^{\alpha} X_{\alpha}=0
$$


and

$$
\nabla_{X_{3}} X_{3}=\sum_{\alpha=1}^{3} \tilde{\Gamma}_{33}^{\alpha} X_{\alpha}=0
$$

Thus $\nabla_{X_{2}} X_{2}$ and $\nabla_{X_{3}} X_{3}$ trivially take their values in $Y$, showing that $X_{2}$ and $X_{3}$ are indeed decoupling vector fields. Next we show that the snakeboard is kinematically controllable by showing that the involutive closure of $\left\{X_{2}, X_{3}\right\}$ has maximal rank. This will follow if and only if the involutive closure of $\left\{c_{1} X_{2}, X_{3}\right\}$ has maximal rank, with $c_{1}$ the function defined in (2.2). We compute

$$
\begin{gathered}
{\left[c_{1} X_{2}, X_{3}\right]=-J_{r} \ell \cos (2 \phi) V_{x}+J_{r} \sin (2 \phi) \frac{\partial}{\partial \theta}-\left(J+J_{r}+J_{w}-m \ell^{2}\right) \sin (2 \phi) \frac{\partial}{\partial \psi}} \\
{\left[X_{3},\left[c_{1} X_{2}, X_{3}\right]\right]=4 J_{r} \ell \sin \phi \cos \phi V_{x}+2 J_{r} \cos (2 \phi) \frac{\partial}{\partial \theta}-2\left(J+J_{r}+J_{w}-m \ell^{2}\right) \frac{\partial}{\partial \psi}} \\
{\left[c_{1} X_{2},\left[X_{3},\left[c_{1} X_{2}, X_{3}\right]\right]\right]=-J_{r}^{2} \ell \sin (2 \phi) V_{y}} \\
{\left[X_{3},\left[c_{1} X_{2},\left[X_{3},\left[c_{1} X_{2}, X_{3}\right]\right]\right]\right]=-2 J_{r}^{2} \ell \cos (2 \phi) V_{y} .}
\end{gathered}
$$

Assembling the components of the vector fields

$$
\left\{c_{1} X_{2}, X_{3},\left[c_{1} X_{2}, X_{3}\right],\left[X_{3},\left[c_{1} X_{2}, X_{3}\right]\right],\left[X_{3},\left[c_{1} X_{2},\left[X_{3},\left[c_{1} X_{2}, X_{3}\right]\right]\right]\right]\right\}
$$

into the columns of a matrix, we determine the determinant of this matrix to be

$$
-4 J_{r}^{4} m \ell^{4} \cos (2 \phi) .
$$

We see that this determinant is nonzero for $\phi \notin\left\{ \pm \frac{\pi}{4}, \pm \frac{3 \pi}{4}\right\}$. At these degenerate values of $\phi$, the vector fields

$$
\left\{c_{1} X_{2}, X_{3},\left[c_{1} X_{2}, X_{3}\right],\left[X_{3},\left[c_{1} X_{2}, X_{3}\right]\right],\left[c_{1} X_{2},\left[X_{3},\left[c_{1} X_{2}, X_{3}\right]\right]\right]\right\}
$$

span the tangent space. This shows that the system is indeed kinematically controllable

\section{Motion primitives for the snakeboard}

We now use the fact that the snakeboard is kinematically controllable using the decoupling vector fields $\left\{X_{2}, X_{3}\right\}$ to pose a nonlinear inversion problem which will solve the motion planning problem for the snakeboard. We also provide an explicit solution to this problem. However, the explicit solution we provide will typically involve an unnecessarily large number of moves for the snakeboard. While we do not propose a systematic methodology to obtain satisfactory solutions to the motion planning problem, we do exhibit an ad hoc solution that demonstrates the potential value of solving the nonlinear inversion problem. We note that our reduction of the problem to a static, low-dimensionat problem presents dynamic model, and perform optimisation on this model. Indeed, the ad hoc solution of problem that we determine is in closed form.

We first make a few remarks that allow us to restrict our interest to the control of the variables $(x, y, \theta)$ while ignoring $(\psi, \phi)$. To do this, we will illustrate how, when at rest in a configuration $\left(x, y, \theta, \psi_{0}, \phi_{0}\right)$, we can steer to $\left(x, y, \theta, \psi_{1}, \phi_{1}\right)$ for any $\psi_{1}$ and $\phi_{1}$. Clearly one can adjust $\phi$ in any way one wants without altering any of the other variables. To alter $\psi$, one can change $\phi$ to zero. In this configuration one can now change $\psi$ to the desired position without affecting the other configuration variables. In this way we reduce our interest to achieving the desired values for $(x, y, \theta) \in S E(2)$. We do this by designing controls that steer from $(0,0,0)$ to points of the form $\left(x_{d}, 0,0\right),\left(0, y_{d}, 0\right)$, and $\left(0,0, \theta_{d}\right)$. The latter motion is obtained along with motion incurred in $x$ and $y$, but this can be corrected using the already obtained $x / y$-translations. By concatenating such motions, starting and ending at rest for each segment, we can achieve any desired configuration of the snakeboard.

4.1. The basic primitive. Movement along the $X_{3}$ decoupling vector field is trivial; it merely specifies a rotation of the wheel angle $\phi$ without altering any of the other configurations. However, movement along the $X_{2}$ decoupling vector field is not so simple, and it is this motion which lies at the heart of our trajectory generation algorithm. Note that the components of $X_{2}$ depend on $\phi$. Therefore, to specify a motion along $X_{2}$, one should first specify a wheel angle $\phi=\phi_{0}$. The only other parameter in a movement along $X_{3}$ will then be the total time taken by the motion. Note that since the coefficient of $\frac{\partial}{\partial x}$ is 1 in $X_{2}$, the time of the manoeuvre will be the change in the rotor angle $\Delta \psi$. What we shall determine is the relationship between $\left(\phi_{0}, \Delta \psi\right)$ and the motion in $(x, y, \theta) \in S E(2)$. To state this relationship, we utilise the definitions of $a(\phi)$ and $b(\phi)$ as given in (2.3). The reader may also wish to recall that, as a matrix group, $S E(2)$ consists of those matrices of the form

$$
\left[\begin{array}{ccc}
\cos \theta & -\sin \theta & x \\
\sin \theta & \cos \theta & y \\
0 & 0 & 1
\end{array}\right], \quad x, y, \theta \in \mathbb{R} .
$$

Furthermore, the Lie algebra of this group consists of those matrices of the form

$$
\left[\begin{array}{ccc}
0 & -\omega & \xi \\
\omega & 0 & \eta \\
0 & 0 & 0
\end{array}\right], \quad \xi, \eta, \omega \in \mathbb{R}
$$

and we denote the collection of all such matrices by $\mathfrak{s e}(2)$. The Lie group exponential coincides with the matrix exponential, and is given in this case by

$$
\exp \left(\left[\begin{array}{ccc}
0 & -\omega & \xi \\
\omega & 0 & \eta \\
0 & 0 & 0
\end{array}\right]\right)=\left[\begin{array}{ccc}
\cos \omega & -\sin \omega & \frac{\sin \omega}{\omega} \xi-\frac{1-\cos \omega}{\omega} \eta \\
\sin \omega & \cos \omega & \frac{1-\cos \omega}{\omega} \xi+\frac{\sin \omega}{\omega} \eta \\
0 & 0 & 1
\end{array}\right]
$$

when $\omega \neq 0$. With this notation, we have the following result.

4.1 Lemma: Let $q_{0}=\left(0,0,0,0, \phi_{0}\right) \in Q$ and let $\left(x, y, \theta, \Delta \psi, \phi_{0}\right) \in Q$ be the point obtained by flowing along $X_{2}$ for time $\Delta \psi$. Then

$$
\begin{aligned}
{\left[\begin{array}{ccc}
\cos \theta & -\sin \theta & x \\
\sin \theta & \cos \theta & y \\
0 & 0 & 1
\end{array}\right] } & =\exp \left(\Delta \psi\left[\begin{array}{ccc}
0 & b\left(\phi_{0}\right) & a\left(\phi_{0}\right) \\
-b\left(\phi_{0}\right) & 0 & 0 \\
0 & 0 & 0
\end{array}\right]\right) \\
& =\left[\begin{array}{ccc}
\left.\cos \left(b\left(\phi_{0}\right) \Delta \psi\right)\right) & \sin \left(b\left(\phi_{0}\right) \Delta \psi\right) & \ell \cot \phi_{0} \sin \left(b\left(\phi_{0}\right) \Delta \psi\right) \\
-\sin \left(b\left(\phi_{0}\right) \Delta \psi\right) & \cos \left(b\left(\phi_{0}\right) \Delta \psi\right) & \ell \cot \phi_{0}\left(\cos \left(b\left(\phi_{0}\right) \Delta \psi\right)-1\right) \\
0 & 0 & 1
\end{array}\right] .
\end{aligned}
$$


Proof: This is simply a matter of explicitly solving the differential equation associated with $X_{2}$ with $\phi$ specified as $\phi_{0}$, and for time $\Delta \psi$.

It is evident from the definition of $X_{2}$ that during a motion along $X_{2}$, one should have $\phi_{0} \notin\{0, \pi\}$ since in such a configuration the rotor will simply move without changing $(x, y, \theta)$. The following lemma describes the possible values of the quantities $a(\phi) \Delta \psi$ and $b(\phi) \Delta \psi$ obtainable using wheel angles between $-\frac{\pi}{2}$ and $\frac{\pi}{2}$.

4.2 LemMA: Let $S, T \subset \mathbb{R}^{2}$ be given by

$$
\begin{aligned}
& S=(]-\frac{\pi}{2}, 0[\cup] 0, \frac{\pi}{2}[) \times(\mathbb{R} \backslash\{0\}) \\
& T=\mathbb{R}^{2} \backslash(\{(x, 0) \mid x \in \mathbb{R}\} \cup\{(0, y) \mid y \in \mathbb{R}\}) .
\end{aligned}
$$

The map $(\phi, \Delta \psi) \mapsto(a(\phi) \Delta \psi,-b(\phi) \Delta \psi)$ is a diffeomorphism from $S$ to $T$.

Proof: The differentiability of the stated map is clear. We can also produce an explicit inverse for the map:

$$
(\xi, \omega) \mapsto\left(-\arctan \left(\frac{\omega \ell}{\xi}\right),-\frac{c_{1}\left(\arctan \left(\frac{\omega \ell}{\xi}\right)\right)\left(\xi^{2}+\ell^{2} \omega^{2}\right)}{J_{r} \ell^{2} \omega}\right) .
$$

This map is itself obviously differentiable on the specified domain.

Combined, Lemmas 4.1 and 4.2 suggest that we should try to do motion planning in the coordinates $(x, y, \theta)$ using as parameters the forward and angular velocities $(\xi, \omega)=$ $\left(a\left(\phi_{0}\right) \Delta \psi, b\left(\phi_{0}\right) \Delta \psi\right)$ constrained to take values in the set $T$. For a physical snakeboard, it is reasonable to suppose that there will be restrictions on $\psi$ and $\phi$. For this reason, we make the assumption that $\psi \in[-\bar{\psi}, \bar{\psi}]$ and $\phi \in[-\bar{\phi}, \bar{\phi}]$ for some $\bar{\phi} \in] 0, \frac{\pi}{2}[$. This provides a restriction that $|\Delta \psi| \leq 2 \psi$. This then defines a set

$$
\bar{S}=(]-\bar{\phi}, 0[\cup] 0, \bar{\phi}[) \times(]-2 \bar{\psi}, 2 \bar{\psi}[),
$$

and the map of Lemma 4.2, when restricted to this set, will have an image as shown in Figure 3 . The angle $\chi$ in the figure is given by $\chi=-\arctan \left(\frac{\ell b(\bar{\phi})}{a(\phi)}\right)$. Let us denote by $\bar{T}$ the region of $\mathbb{R}^{2}$ shown in Figure 3 .

4.2. A nonlinear inversion problem for snakeboard motion planning, and an explicit solution. The idea of kinematic controllability is that one follows concatenations of integral curves of decoupling vector fields, ensuring that at the beginning and end of each segment, one is at rest. With the discussion of the preceding section as background, we can state the essence of the motion planning problem for the snakeboard as follows.

4.3 SNakeboard motion PlanNing Problem: Suppose that $q_{\mathrm{i}}=\left(x_{\mathrm{i}}, y_{\mathrm{i}}, \theta_{\mathrm{i}}, \psi_{\mathrm{i}}, \phi_{\mathrm{i}}\right)$ and $q_{\mathrm{f}}=\left(x_{\mathrm{f}}, y_{\mathrm{f}}, \theta_{\mathrm{f}}, \psi_{\mathrm{f}}, \phi_{\mathrm{f}}\right)$ are given. Find a finite collection of points $\left(\xi_{1}, \omega_{1}\right), \ldots,\left(\xi_{k}, \omega_{k}\right) \in$ $\bar{T}$ so that

$$
\begin{array}{r}
{\left[\begin{array}{ccc}
\cos \theta_{\mathrm{i}} & -\sin \theta_{\mathrm{i}} & x_{\mathrm{i}} \\
\sin \theta_{\mathrm{i}} & \cos \theta_{\mathrm{i}} & y_{\mathrm{i}} \\
0 & 0 & 1
\end{array}\right] \circ \exp \left(\left[\begin{array}{ccc}
0 & -\omega_{1} & \xi_{1} \\
\omega_{1} & 0 & 0 \\
0 & 0 & 0
\end{array}\right]\right) \circ \cdots \circ \exp \left(\left[\begin{array}{ccc}
0 & -\omega_{k} & \xi_{k} \\
\omega_{k} & 0 & 0 \\
0 & 0 & 0
\end{array}\right]\right)=} \\
{\left[\begin{array}{ccc}
\cos \theta_{\mathrm{f}} & -\sin \theta_{\mathrm{f}} & x_{\mathrm{f}} \\
\sin \theta_{\mathrm{f}} & \cos \theta_{\mathrm{f}} & y_{\mathrm{f}} \\
0 & 0 & 1
\end{array}\right]}
\end{array}
$$

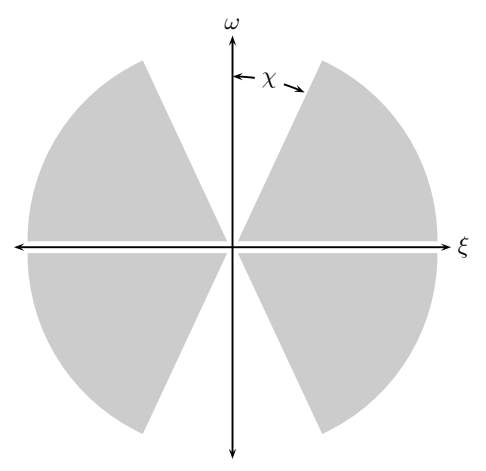

FIGURE 3. The gray region describes the set of admissible values for the forward and angular velocities $(\xi, \omega)$ with constraints on the wheel and rotor angles

Of course, in obtaining a solution to the problem, one will want to minimise $k$. In the absence of control constraints, it is possible to show that one can steer from an initial configuration to any final configuration with $k$ at most 3 . However, it appears quite difficult to analytically obtain such bounds in the presence of constraints on the rotor and wheel angles.

Let us now turn to exhibiting an explicit local solution to Problem 4.3. That is, for $\left(x_{\mathrm{f}}, y_{\mathrm{f}}, \theta_{\mathrm{f}}\right)$ sufficiently close to $(0,0,0)$, we shall show how to steer from $(0,0,0)$ to the final point using a finite sequence of primitives. The strategy is first to show how sufficiently small manoeuvres in the body $\xi$ and $\eta$-directions (see Figure 2) can be performed while leaving $\theta$ unchanged. Then we demonstrate how one may achieve a desired change in the angle $\theta$, but with a translation by some determinable amount in $x$ and $y$. Using the previously determined motions in $\xi$ and $\eta$, one can then achieve a change in $\theta$ while leaving $x$ and $y$ unchanged. This gives rise to a complete closed-form local planner. It would be possible to design a global planner by computing appropriate sequences of desired local displacements and composing multiple invocations of the local planner. 
$\xi$-translation

One can determine that for a fixed $\phi_{0}, \Delta \psi>0$ we construct a sequence of three basic primitives as follows:

$$
\begin{gathered}
\exp \left(\Delta \psi\left[\begin{array}{ccc}
0 & b\left(\phi_{0}\right) & a\left(\phi_{0}\right) \\
-b\left(\phi_{0}\right) & 0 & 0 \\
0 & 0 & 0
\end{array}\right]\right) \circ \exp \left(-2 \Delta \psi\left[\begin{array}{ccc}
0 & b\left(-\phi_{0}\right) & a\left(-\phi_{0}\right) \\
-b\left(-\phi_{0}\right) & 0 & 0 \\
0 & 0 & 0
\end{array}\right]\right) \circ \\
\exp \left(\Delta \psi\left[\begin{array}{ccc}
0 & b\left(\phi_{0}\right) & a\left(\phi_{0}\right) \\
-b\left(\phi_{0}\right) & 0 & 0 \\
0 & 0 & 0
\end{array}\right]\right)=\left[\begin{array}{ccc}
1 & 0 & \Delta \xi \\
0 & 1 & 0 \\
0 & 0 & 1
\end{array}\right],
\end{gathered}
$$

where

$$
\Delta \xi=4 \ell \cot \phi_{0} \sin \left(\frac{J_{r} \Delta \psi \sin ^{2} \phi_{0}}{c_{1}\left(\phi_{0}\right)}\right) .
$$

Thus we can, for a fixed $\phi_{0}$ and for $\Delta \xi$ sufficiently small, explicitly choose $\Delta \psi$ to translate the snakeboard in the body $\xi$-direction by an amount $\Delta \xi$. In practice, the amount of the translation can be reasonably large. To obtain arbitrarily large translations, one can of course compose smaller ones as we have constructed here.

$\eta$-translation

The story here is much the same as for the $\xi$-translation, except that we use the following sequence of four basic primitives:

$$
\begin{gathered}
\exp \left(\Delta \psi\left[\begin{array}{ccc}
0 & b\left(\phi_{0}\right) & a\left(\phi_{0}\right) \\
-b\left(\phi_{0}\right) & 0 & 0 \\
0 & 0 & 0
\end{array}\right]\right) \circ \exp \left(-\Delta \psi\left[\begin{array}{ccc}
0 & b\left(-\phi_{0}\right) & a\left(-\phi_{0}\right) \\
-b\left(-\phi_{0}\right) & 0 & 0 \\
0 & 0 & 0
\end{array}\right]\right) \circ \\
\exp \left(-\Delta \psi\left[\begin{array}{ccc}
0 & b\left(\phi_{0}\right) & a\left(\phi_{0}\right) \\
-b\left(\phi_{0}\right) & 0 & 0 \\
0 & 0 & 0
\end{array}\right]\right) \circ \exp \left(\Delta \psi\left[\begin{array}{ccc}
0 & b\left(-\phi_{0}\right) & a\left(-\phi_{0}\right) \\
-b\left(-\phi_{0}\right) & 0 & 0 \\
0 & 0 & 0
\end{array}\right]\right)= \\
{\left[\begin{array}{ccc}
1 & 0 & 0 \\
0 & 1 & \Delta \eta \\
0 & 0 & 1
\end{array}\right],}
\end{gathered}
$$

where

$$
\Delta \eta=4 \ell \cot \phi_{0}\left(\cos \left(\frac{J_{r} \Delta \psi \sin ^{2} \phi_{0}}{c_{1}\left(\phi_{0}\right)}\right)-1\right) .
$$

Thus for $\Delta \eta$ sufficiently small for a chosen $\phi_{0}$, one can achieve a pure translation in the body $\eta$-direction. In practice, and not surprisingly, to move in the $\eta$-direction is more difficult that the corresponding $\xi$-translations. Nevertheless, one can still compose sequences of small $\eta$-translations to achieve any specified motion in that direction.

\section{$\theta$-specification}

Unlike our translations in $x$ and $y$, the motion in the $\theta$-direction is achieved while incurring motion in the other variables as well. The single primitive we use is

$$
\exp \left(\Delta \psi\left[\begin{array}{ccc}
0 & b\left(\phi_{0}\right) & a\left(\phi_{0}\right) \\
-b\left(\phi_{0}\right) & 0 & 0 \\
0 & 0 & 0
\end{array}\right]\right)=\left[\begin{array}{ccc}
\cos (\Delta \theta) & -\sin (\Delta \theta) & \Delta x \\
\sin (\Delta \theta) & \cos (\Delta \theta) & \Delta y \\
0 & 0 & 1
\end{array}\right],
$$

where

$$
\begin{aligned}
& \Delta \theta=-\frac{J_{r} \Delta \psi \sin ^{2} \phi_{0}}{c_{1}\left(\phi_{0}\right)} \\
& \Delta x=\ell \cot \phi_{0} \sin \left(\frac{J_{r} \Delta \psi \sin ^{2} \phi_{0}}{c_{1}\left(\phi_{0}\right)}\right) \\
& \Delta y=\ell \cot \phi_{0}\left(\cos \left(\frac{J_{r} \Delta \psi \sin ^{2} \phi_{0}}{c_{1}\left(\phi_{0}\right)}\right)-1\right) .
\end{aligned}
$$

Although there is an error in $x$ and $y$-translations, these can of course be corrected by the employment of the direct translations in these directions.

In Figure 4 we show a motion of the snakeboard from $(x, y, \theta)=(0,0,0)$ to $(x, y, \theta)=$ $\left(\sqrt{2}, 2, \frac{\pi}{5}\right)$. The rotation by $\frac{\pi}{5}$ was broken into two smaller rotations to mollify the effects of the deviation in $x$ and $y$. We note that, including final corrections to the wheel and rotor angles, the above sort of motion will involve a concatenation of twenty basic kinematic motions. While some of these twenty motions are very simple, it is clear that this is generally a rather inefficient way of generating snakeboard motion.

An ad hoc solution to the nonlinear inversion problem

The above procedure demonstrates an explicit solution to Problem 4.3, albeit a rather inefficient one. One can also proceed by looking directly at the equations yielded by Problem 4.3. Without the constraints on the wheel and rotor angles, it is possible to construct a procedure that will steer the snakeboard between two $(x, y, \theta)$ configurations using a concatenation of at most three basic primitives. One can do this by looking at the equations generated by the problem, noting that for three primitives there will be six independent variables (three pairs $(\xi, \omega)$ ). One can then impose relations on these variables to reduce the extent to which the problem is under-determined. In this way, various sorts of ad hoc procedures are fairly easily developed. However, it appears to be difficult to do this in a methodical way so that the wheel and rotor angle constraints are satisfied. In Figure 5 we show such a three primitive motion.

4.4 Remarks: 1. For the purposes of making more easily understood plots, we have ignored constraints on the rotor angle $\psi$. When constraints on this angle are enforced, the motions become comparatively smaller than those in Figures 4 and 5.

2. The controls which provide the motions shown in Figures 4 and 5 are simple in form, being merely cosine's with an easily represented amplitude and frequency.

3. For an accurate comparison of the motion in Figure 5 with the motion of Figure 4 , we should also count the motions involving positioning the wheels, and straightening 

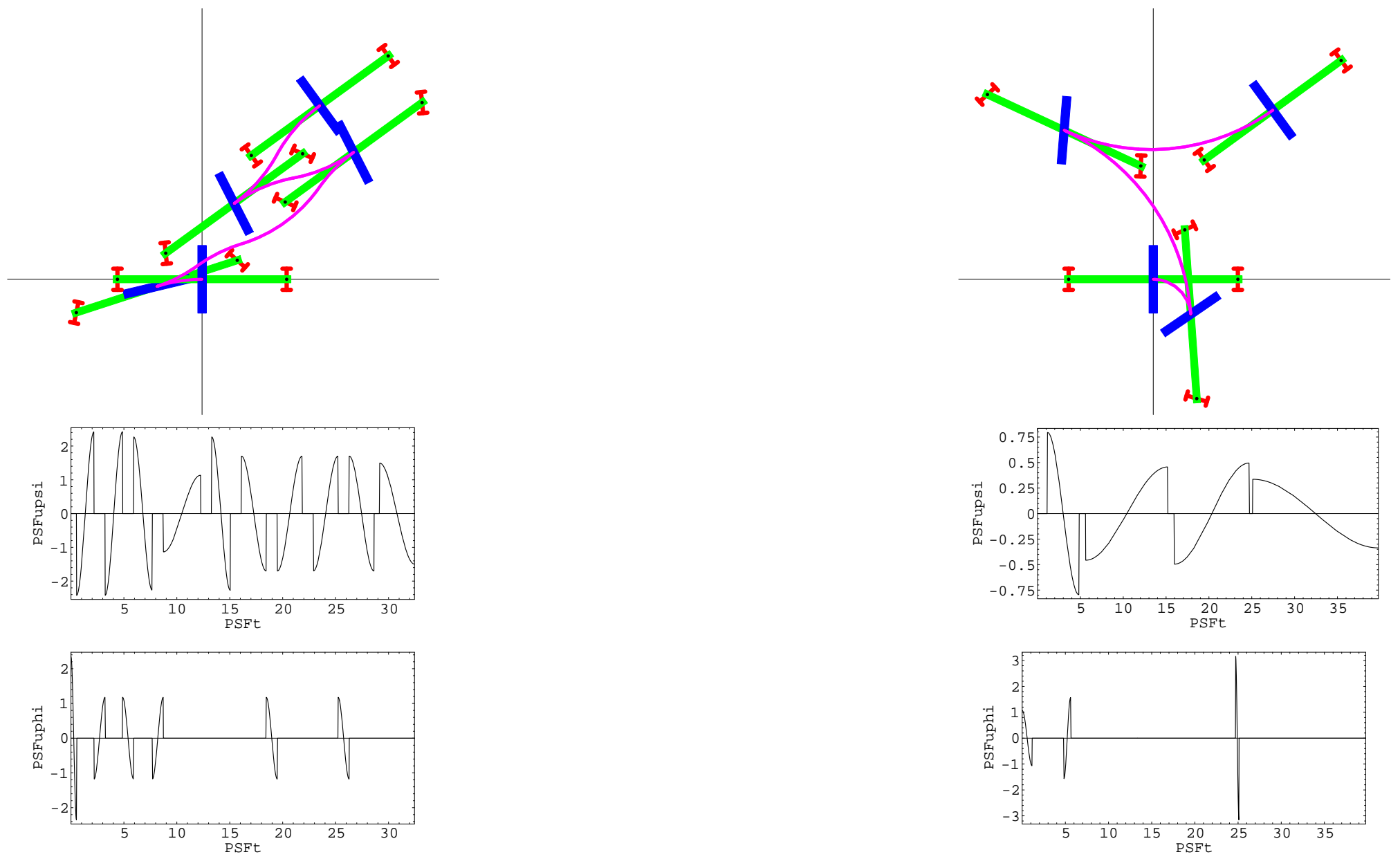

FIGURE 4. Snakeboard motion from $(0,0,0)$ to $\left(\sqrt{2}, 2, \frac{\pi}{5}\right)$ using concatenations of basis primitives. The controls are shown in
the bottom figure. The parameters used are $\left(m, \ell, J, J_{r}, J_{w}\right)=$ $\left(1,1,1,1, \frac{1}{4}\right)$.

Figure 5. Snakeboard motion from $(0,0,0)$ to $\left(\sqrt{2}, 2, \frac{\pi}{5}\right)$ using an ad hoc solution to Problem 4.3. The controls are shown in the bottom figure. The parameters used are $\left(m, \ell, J, J_{r}, J_{w}\right)=$ $\left(1,1,1,1, \frac{1}{4}\right)$. 
out the rotor and wheels after the motion. In this case, there are eight segments of the motion in Figure 5, compared with twenty for the motion in Figure 4.

4. Interestingly, however, the time taken for the motions in Figures 4 and 5 are comparable.

5. Also, by simple tweaking it is possible to generate rather larger motions using ad hoc methods than by using the systematic concatenation of trajectories using $(x, y, \theta)$ translations.

\section{Discussion}

The snakeboard is a somewhat simple example of a mechanical system, and yet it is certainly not trivial. Indeed, until now, the motion planning problem for the snakeboard has resisted any sort of non-numerical solution. In this paper we have shown that it is possible to reduce the motion planning problem for the snakeboard to a low-dimensional static nonlinear inversion problem. Although this problem is not of particularly pleasing nature, we have shown that it admits solution by explicitly demonstrating a simple local motion planning strategy for the snakeboard in closed form.

The essential point to be made here is that it is the affine connection formalism which lies at the heart of the proposed solution to the motion planning problem. Indeed, it is in this setting that the notions of decoupling vector fields and kinematic controllability are naturally understood. It is also worth noting that the idea of decoupling vector fields arises naturally in the quadratic form controllability conditions of Hirschorn and Lewis [2001]. This promising and nontrivial intertwining of controllability conditions and motion planning algorithms is no doubt a promising avenue for further research.

\section{References}

Bloch, A. M. and Crouch, P. E. [1998] Newton's law and integrability of nonholonomic systems, SIAM Journal on Control and Optimization, 36(6), 2020-2039.

Bloch, A. M., Krishnaprasad, P. S., Marsden, J. E., and Murray, R. M. [1996] Nonholonomic mechanical systems with symmetry, Archive for Rational Mechanics and Analysis, 136(1), $21-99$.

Bullo, F. and Lynch, K. M. [2001] Kinematic controllability and decoupled trajectory planning for underactuated mechanical systems, Institute of Electrical and Electronics Engineers. Transactions on Robotics and Automation, 17(4), 402-412.

Bullo, F. and Žefran, M. [2002] On mechanical systems with nonholonomic constraints and symmetries, Systems \& Control Letters, 45(2), 133-143.

Cortés, J., Martínez, S., and Bullo, F. [2002] On nonlinear controllability and series expansions for Lagrangian systems with dissipative forces, Institute of Electrical and Electronics Engineers. Transactions on Automatic Control, 47(8), 1396-1401.
Hirschorn, R. M. and Lewis, A. D. [2001] Geometric first-order controllability conditions for affine connection control systems, in Proceedings of the 40th IEEE Conference on Decision and Control, pages 4216-4221, Institute of Electrical and Electronics Engineers, Orlando, FL.

Lewis, A. D. [1998] Affine connections and distributions with applications to nonholonomic mechanics, Reports on Mathematical Physics, 42(1/2), 135-164.

- [1999] When is a mechanical control system kinematic?, in Proceedings of the 38th IEEE Conference on Decision and Control, pages 1162-1167, Institute of Electrical and Electronics Engineers, Phoenix, AZ.

- [2000] Simple mechanical control systems with constraints, Institute of Electrical and Electronics Engineers. Transactions on Automatic Control, 45(8), 1420-1436.

Lewis, A. D., Ostrowski, J. P., Murray, R. M., and Burdick, J. W. [1994] Nonholonomic mechanics and locomotion: the Snakeboard example, in Proceedings of the IEEE International Conference on Robotics and Automation, pages 2391-2400, Institute of Electrical and Electronics Engineers, San Diego.

Lynch, K. M., Shiroma, N., Arai, H., and Tanie, K. [2000] Collision-free trajectory planning for a 3-DOF robot with a passive joint, The International Journal of Robotics Research, 19(12), 1171-1184.

Ostrowski, J. P. [1995] The Mechanics and Control of Undulatory Robotic Locomotion, Ph.D. thesis, California Institute of Technology, Pasadena, California, USA. URL: http://www.cds.caltech.edu/

- [2000] Steering for a class of dynamic nonholonomic systems, Institute of Electrical and Electronics Engineers. Transactions on Automatic Control, 45(8), 1492-1497.

Ostrowski, J. P. and Burdick, J. W. [1997] Controllability tests for mechanical systems with constraints and symmetries, Journal of Applied Mathematics and Computer Science, $\mathbf{7}(2), 101-127$.

Ostrowski, J. P., Desai, J. P., and Kumar, V. [2000] Optimal gait selection for nonholonomic locomotion systems, The International Journal of Robotics Research, 19(5), 225-237.

Synge, J. L. [1928] Geodesics in nonholonomic geometry, Mathematische Annalen, 99, 738- 